\title{
Producing ultrashort Terahertz to UV photons at high repetition rates for research into materials
}

G. R. Neil, C. Behre, S. V. Benson, G. Biallas, J. Boyce, L.A. Dillon-Townes, D. Douglas, H. F. Dylla, R. Evans, A. Grippo, D. Gruber, J. Gubeli, C. Hernandez-Garcia, K. Jordan, M. J. Kelley, L. Merminga, J. Mammosser, N. Nishimori, J. Preble, R. Rimmer, M. Shinn, T. Siggins, R. Walker, G. P. Williams, and S. Zhang

Thomas Jefferson National Accelerator Facility

Newport News VA 23606 USA

\section{ABSTRACT}

A new THz/IR/UV photon source at Jefferson Lab is the first of a new generation of light sources based on a Energy-Recovered, (superconducting) Linac (ERL). The machine has a $160 \mathrm{MeV}$ electron beam and an average current of $10 \mathrm{~mA}$ in $75 \mathrm{MHz}$ repetition rate hundred femtosecond bunches.

These electron bunches pass through a magnetic chicane and therefore emit synchrotron radiation. For wavelengths longer than the electron bunch the electrons radiate coherently a broadband $\mathrm{THz} \sim$ half cycle pulse whose average brightness is $>5$ orders of magnitude higher than synchrotron IR sources. Previous measurements showed $20 \mathrm{~W}$ of average power extracted[1]. The new facility offers simultaneous synchrotron light from the visible through the FIR along with broadband THz production of 100 fs pulses with $>200 \mathrm{~W}$ of average power (see G. P. Williams, this conference).

The FELs also provide record-breaking laser power [2]: up to $10 \mathrm{~kW}$ of average power in the IR from 1 to 14 microns in 400 fs pulses at up to $74.85 \mathrm{MHz}$ repetition rates and soon will produce similar pulses of 300-1000 nm light at up to $3 \mathrm{~kW}$ of average power from the UV FEL. These ultrashort pulses are ideal for maximizing the interaction with material surfaces. The optical beams are Gaussian with nearly perfect beam quality. See www.jlab.org/FEL for details of the operating characteristics; a wide variety of pulse train configurations are feasible from 10 microseconds long at high repetition rates to continuous operation.

The THz and IR system has been commissioned. The UV system is to follow in 2005. The light is transported to user laboratories for basic and applied research. Additional lasers synchronized to the FEL are also available. Past activities have included production of carbon nanotubes, studies of vibrational relaxation of interstitial hydrogen in silicon, pulsed laser vapor deposition, nitriding of metals, and energy flow in proteins. This paper will present the status of the system and discuss some of the opportunities provided by this unique light source for modifying and studying materials.

\section{INTRODUCTION}

A Free Electron Laser (FEL) called the IR/UV Upgrade is operational as a user facility at Thomas Jefferson National Accelerator Facility in Newport News, Virginia, USA. Its design is based on an earlier system called the IR Demo which produced over $2 \mathrm{~kW}$ of mode-locked laser power at 3 microns[2]. The electron beam for this was running at $4.5 \mathrm{~mA} \mathrm{CW}$ in a $74.85 \mathrm{MHz}$ train of $60 \mathrm{pC}, 48$ $\mathrm{MeV}$ sub-picosecond pulses. As an evolutionary expansion of the JLab IR Demo FEL [3], the Jefferson Lab Upgrade FEL[4] retains the approach used in the earlier machine - that of a modest gain, high average power, wiggler-driven optical resonator with an energy-recovering SRF linear accelerator

operating at high repetition rate. The $10 \mathrm{~kW}$ design goal is achieved via an increase in both drive beam power (doubled current and quadrupled energy) and FEL extraction efficiency (from $0.5 \%$ to $1 \%$ ). Primary beam specifications and achieved performance for the Upgrade are listed in Table 1. 
Table 1. Upgrade FEL system parameters

\begin{tabular}{lll} 
Driver Accelerator & Design Spec. & Achieved \\
\cline { 2 - 3 } & & 160 \\
Linac Energy & $145 \mathrm{MeV}$ & 9.1 \\
Linac Ave. Current & $10 \mathrm{~mA}$ & 150 \\
Charge & $135 \mathrm{pC}$ & $<15$ \\
Transverse Emittance & $30 \mathrm{~mm}-\mathrm{mrad}$ & 0.3 \\
Energy Spread & $0.3 \%$ & 0.35 \\
Bunch length * & $0.5 \mathrm{ps}$ & $<80$ \\
Longitudinal Emittance & $50 \mathrm{kV}-\mathrm{ps}$ & $74.85 \mathrm{MHz}$ \\
Pulse Repetition Rate & $74.85 \mathrm{MHz}$ & \\
FEL System & & \\
\hline \multirow{2}{*}{ Ave. Power (cw) } & $10 \mathrm{~kW}$ & 8.5 \\
Ave. Power (pulsed) & $\mathrm{n} / \mathrm{a}$ & $10.6 \mathrm{for} 1 \mathrm{~s}$ \\
Operating Wavelengths & $1-14 \mathrm{\mu m}$ & $3,5.5-6.5,10 \mu \mathrm{mm}$ \\
Lasing efficiency & $1 \mathrm{~kW} / \mathrm{mA}$ & 2.6 \\
& & $132 \mathrm{~kW}$ \\
Stored Optical Power (@6um) & $132 \mathrm{~kW}$ & \\
*at desired energy spread & &
\end{tabular}

\section{SOURCE DESCRIPTION}

Figure 1 illustrates the Upgrade design. It comprises a $10 \mathrm{MeV}$ injector, a linac consisting of three Jefferson Lab cryomodules generating a total of 80 to $160 \mathrm{MeV}$ of energy gain, and a recirculator. The latter provides beam transport to, and phase space conditioning of, the accelerated electron beam for the FEL and then returns and prepares the drive beam for energy recovery in the linac.

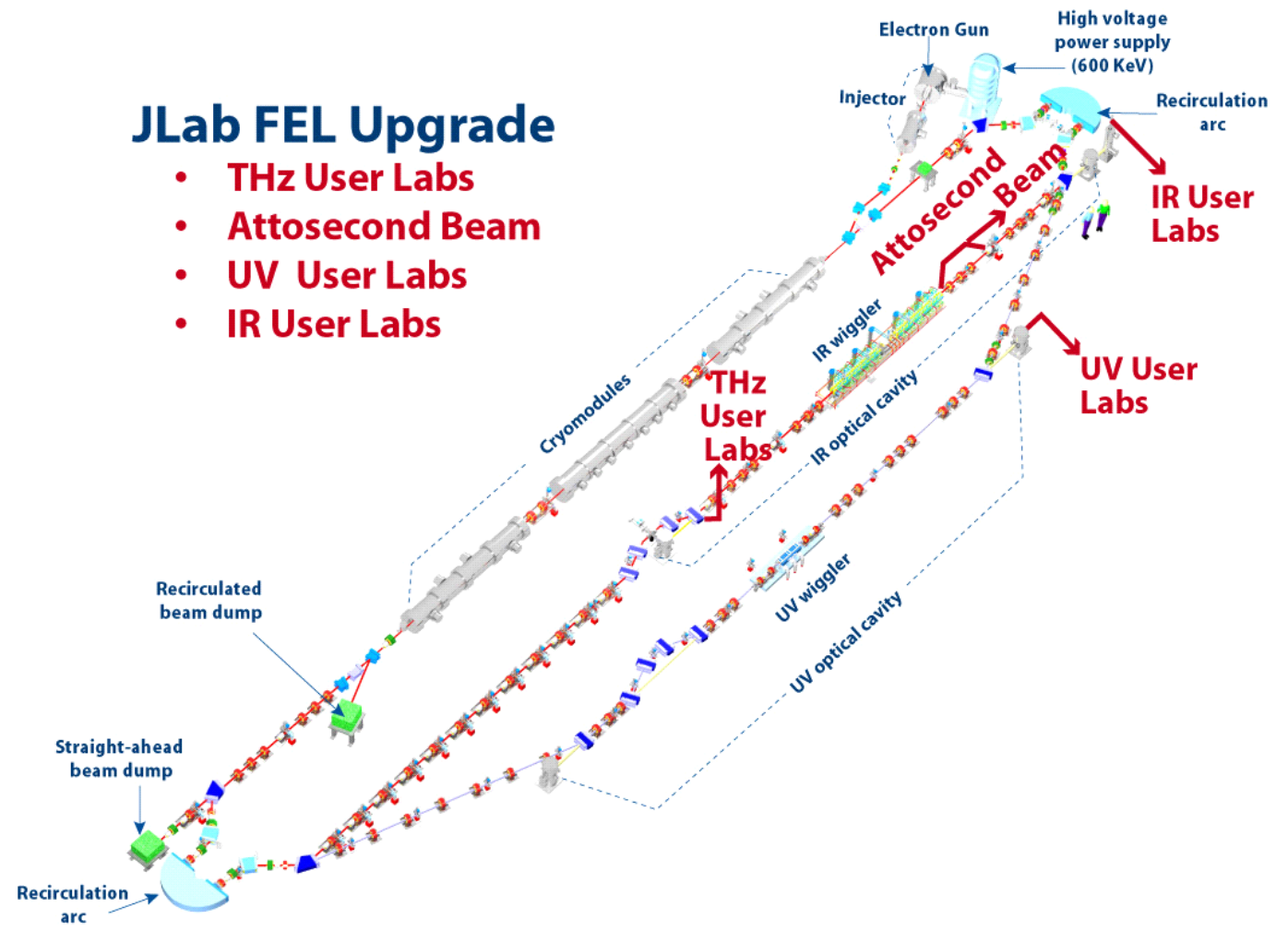

Figure 1: Layout of the IR/UV Upgrade 
The injector is a direct upgrade of the IR Demo injector [5] from $5 \mathrm{~mA}$ to $10 \mathrm{~mA}$ at $10 \mathrm{MeV}$. The current is doubled by an increase of the single bunch charge from $67 \mathrm{pC}$ to $135 \mathrm{pC}$ while maintaining the $75 \mathrm{MHz}$ repetition rate. The injector performance has already met specifications at the elevated bunch charge. Improvements in the voltage standoff capability of the photocathode may allow even brighter source performance in the future.

The linac comprises three cryomodules; the first and third incorporate a conventional five-cell CEBAF cavities, and the central module is based on new seven-cell JLAB cavities [6]. The new module has demonstrated $82 \mathrm{MV}$ of continuous acceleration surpassing all previous such systems. The beam is accelerated (energy recovered) off crest (off trough) so as to impose a phase energy correlation on the longitudinal phase space used in subsequent transport to longitudinally match the beam to the required phase space at the wiggler (dump). That is to say, the bunch is kept relatively long during acceleration, compressed to high peak current just before the wiggler, then temporally expanded before reinsertion into the energy recovery phase of the linac.

The energy recovery transport consists of a second Bates-style end loop followed by a six-quad telescope[7]. The beam is matched to the arc by the second telescope of the FEL insertion; the energy recovery telescope matches beam envelopes from the arc to the linac acceptance. Because energy recovery occurs off-trough, the imposed phase-energy correlations are selected to generate energy compression during energy recovery, yielding a long, low momentum spread bunch at the dump. Measurements indicate that the Upgrade will tolerate an induced energy spread from the FEL of 15\% compressing it to a final spread of order $\pm 1 \%$ - despite the large ratio of final to initial energy. Calculations and measurements show that the emittance growth due to coherent synchrotron radiation (CSR) is not a problem for this design[8].

To provide flexibility and allow initial operation over a range of IR wavelengths, an optical klystron was chosen with two wigglers of 12 periods separated by a dispersion section with 40 periods of path-length delay at the highest wiggler strength. The wiggler period is $20 \mathrm{~cm}$ and the maximum $\mathrm{rms} \mathrm{K}^{2}$ is $>16$. Calculated gain and power are shown in Figure $2 \mathrm{a}$ for operation of the optical klystron as a 25 period wiggler with the UV wiggler performance in $2 \mathrm{~b}$. Both power and gain are very weakly dependent on the emittance and energy spread. A substantial amount of experience was acquired with the system over the last year as presented below. To allow for shorter wavelength operation this wiggler has now been replaced by a wiggler of $8 \mathrm{~cm}$ wavelength with a maximum $\mathrm{K}$ of 1.2. This system will operate in the 1 to 4 micron region and is undergoing commissioning at this time. See Figure 3 for the expected performance of this system.

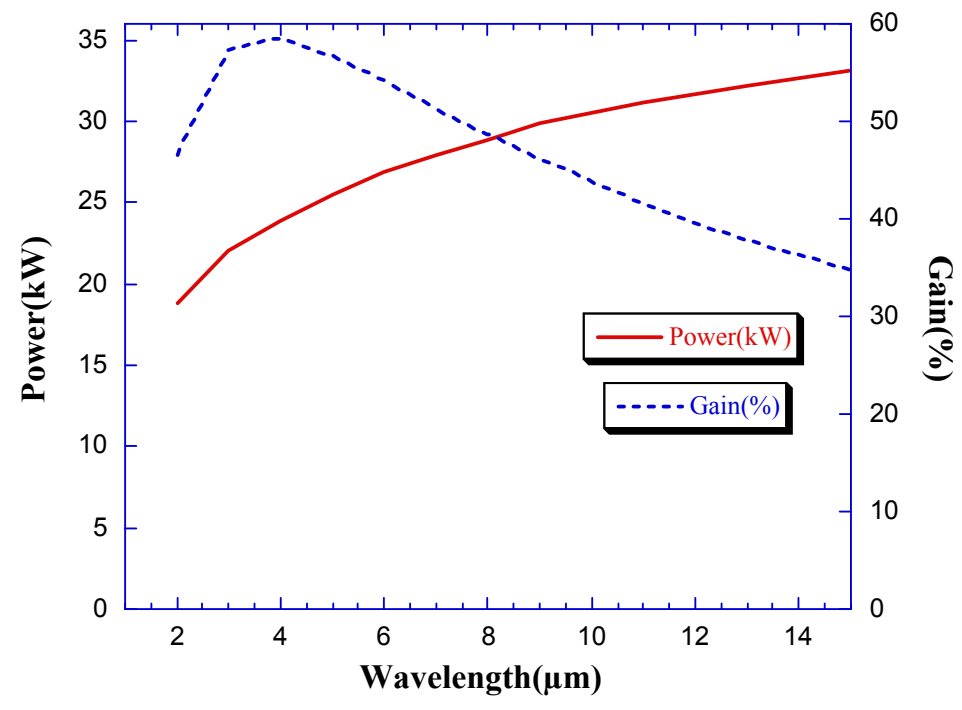




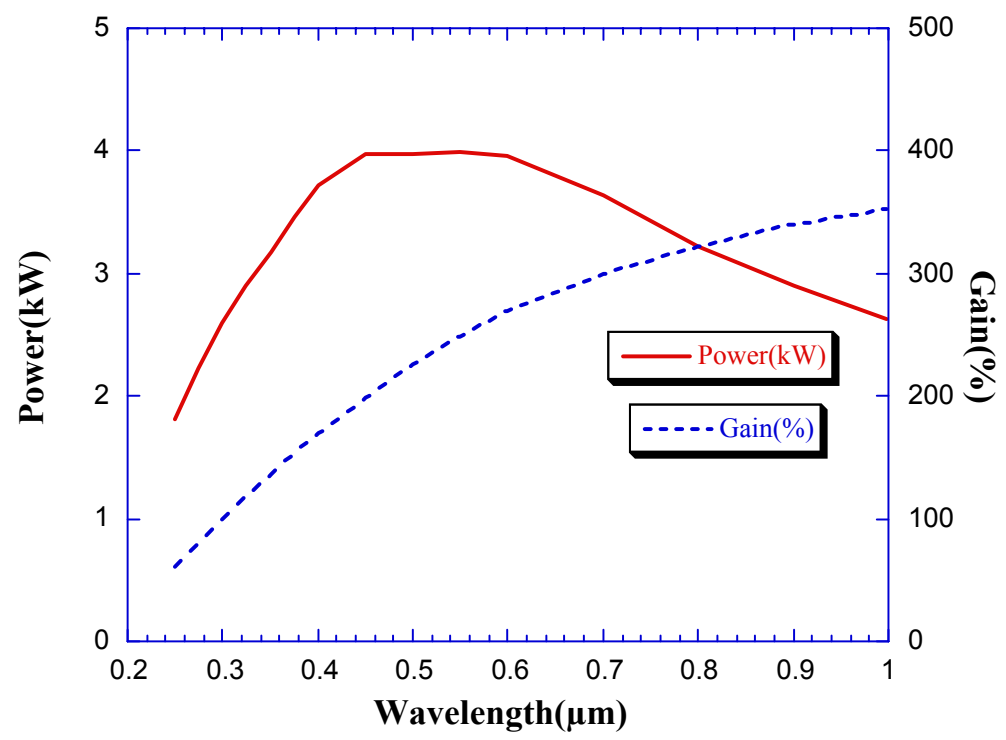

Figure 2. Lasing performance with a) the optical klystron and b) projected UV wiggler

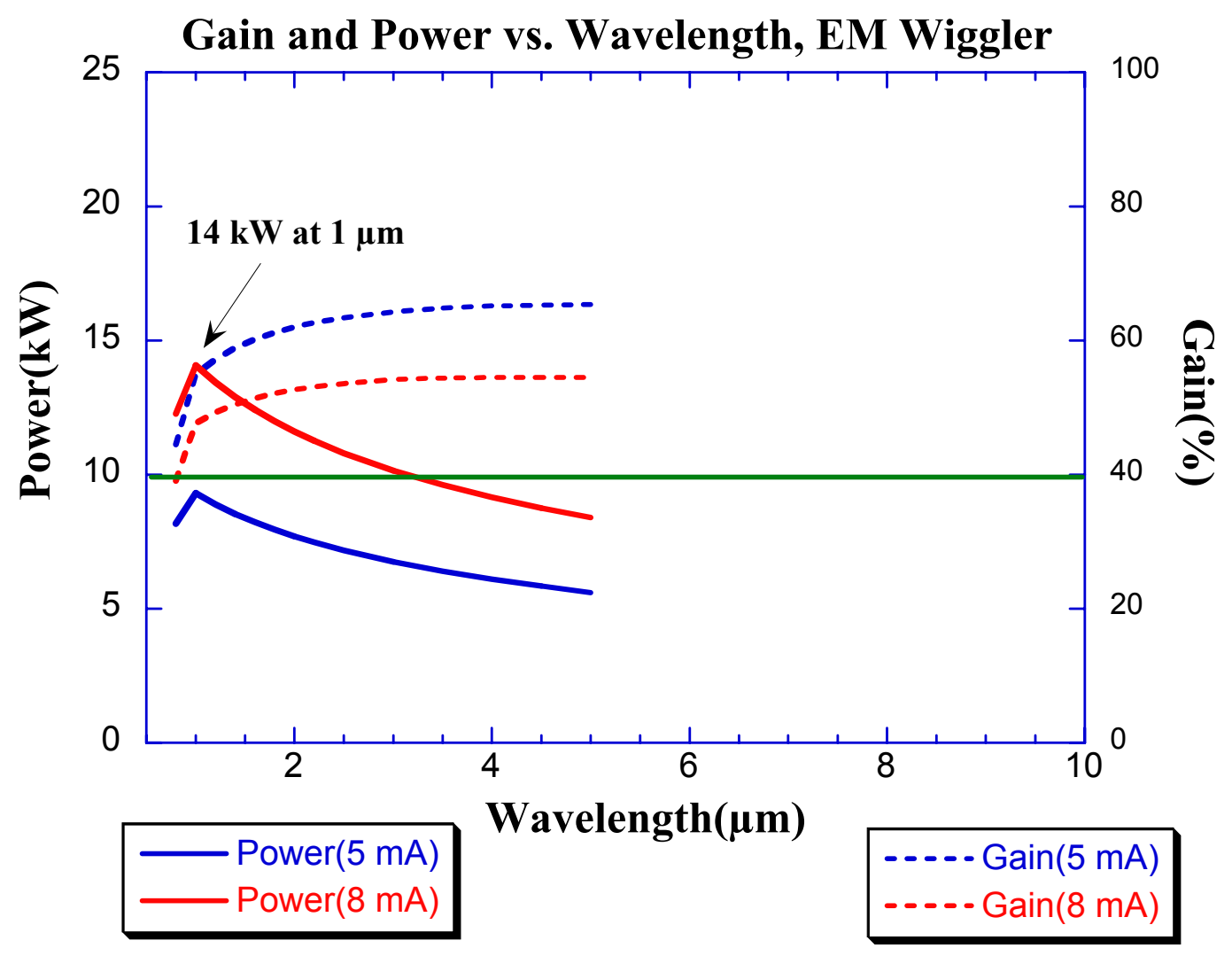

Figure 3. Performance in the IR with the new $8 \mathrm{~cm}$ period electromagnetic wiggler.

The optical resonator is similar to the one used in the IR Demo FEL. To accommodate a longer wiggler, the resonator length has been increased to $32 \mathrm{~m}$. Changes in waist size with mirror heating are 
controlled by using a deformable high reflector mirror. Significant effort was expended to engineer an optical system that would operate under UHV conditions, permit switching of mirrors for coverage of the multiple bands desired, achieve sub-microradian alignment and sub-micron length tolerances, and deal with in excess of $100 \mathrm{~kW}$ of circulating optical power. Our experience in meeting these demanding challenges is discussed below.

For THz production, the electron bunches pass a chicane around the optical cavity, and therefore emit synchrotron radiation. When the electron bunch length approaches that of the wavelength of the light being emitted, the entire bunch of up to 135 picocoulombs of charge $\left(9 \times 10^{8}\right.$ electrons $)$, radiates coherently. The result is a broadband spectrum whose average brightness is more than 5 orders of magnitude higher than can be obtained from conventional incoherent synchrotron IR sources, and 8 orders of magnitude higher than a $2000 \mathrm{~K}$ thermal source. Previous record-breaking measurements showed $20 \mathrm{~W}$ of broadband power extracted in agreement with theory [1]. Figure 4 shows the $\mathrm{THz}$ production from this system. The new facility offers simultaneous synchrotron light from the visible through the FIR along with broadband T-ray (quasi-single-cycle terahertz pulses) production of $100 \mathrm{fs}$ pulses with $>100 \mathrm{~W}$ of average power in a User Facility. See the paper by G. Williams et al. these proceedings for more details of the measurements confirming this performance.

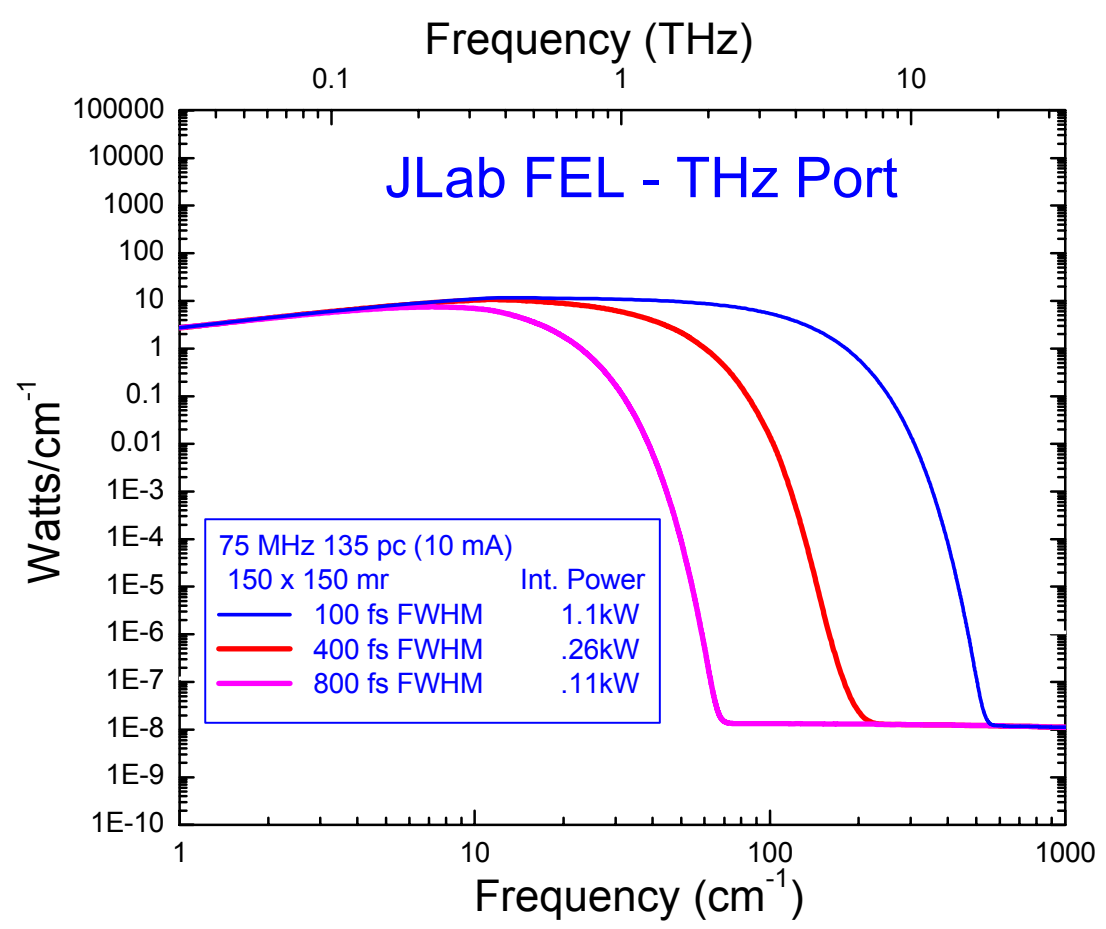

Figure 4. THz radiation production in the IR Upgrade

The THz and IR machine is now essentially complete with the UV beam line to be completed in FY'05. Except for the full average current and the longitudinal emittance, the machine matches the design values. The FEL efficiency closely matched predictions made by pulse propagation codes. A current of $9 \mathrm{~mA}$ has been demonstrated as well as energies up to $160 \mathrm{MeV}$. We are now working on extensions of performance to the 1 micron region from that discussed below.

\section{OPERATIONAL EXPERIENCE}




\section{$\underline{\text { IR Demo performance }}$}

The original IR Demo laser produced up to $2.1 \mathrm{~kW}$ at 3 microns or 150 times the $\mathrm{CW}$ average power of any other FEL in the world and substantially more than any tunable IR laser or subpicosecond laser. The wavelength produced by the FEL was controlled by tuning the electron beam energy but suitable mirrors had to be used for each wavelength band to maximize the power output. The system lased in three primary wavelength bands of 3, 5, and 6 microns dictated by user interest.

In addition to the fundamental output the system produced 4 watts on the fifth harmonic at 1 micron. It produced useful amounts of power at the third harmonic, $300 \mathrm{~W}$ continuously [9] and even conversion of this to green $(\sim 60 \mathrm{~W})$ and $\mathrm{UV}(\sim 15 \mathrm{~W} \mathrm{CW})$ at high efficiency in doubling, tripling and quadrupling crystals. We also lased at 2 microns on the second harmonic producing an interesting $\mathrm{TM}_{02}$ mode structure [10]. Up to $10^{9}$ photons/sec of Thompson scattered X-rays in the 5 to $15 \mathrm{keV}$ range were produced when the FEL pulse scattered off the subsequent electron bunch [11]. Also the system synchronously produced $\sim 50 \mathrm{~W}$ of sub-picosecond $\mathrm{THz}$ pulses from coherent synchrotron emission in a bend.

\section{IR Upgrade performance}

Achieving higher power out of the FEL Upgrade required meeting the new electron beam specifications and then dealing with a number of optical challenges. We met all the key electron beam requirements rather quickly but have had significant challenges in dealing with thermal issues on long wavelength optics. Optical challenges fall into two categories: 1. getting mirror losses down to acceptable levels, and 2. handling the circulating and outcoupled power. We gradually learned how to accomplish both of these.

Previous work has shown that there is a limit to how much power the mirrors can absorb before the power saturates [12]. For example, a zinc selenide output coupler can absorb $5 \mathrm{~W} / \mu \mathrm{m} * \lambda$ of power before the FEL power will saturate. In practice one can slightly exceed this number but it is a good design point. With the first set of $10 \mu \mathrm{m}$ mirrors we found a large amount of mirror heating for even low power. Mirror losses were found to be $0.74 \%$ and $0.3 \%$ for the output coupler (OC) and the high reflector (HR) respectively. We replaced the OC with a mirror with $0.4 \%$ loss. This allowed us to achieve up to $700 \mathrm{~W}$ of CW power but it was clear that $10 \mathrm{~kW}$ was out of reach unless the losses could be reduced to under 500 parts per million (PPM). Discussion with mirror vendors indicated that this was highly unlikely.

From our experience on the IR Demo we knew that lower loss coatings were available in the 6 micron range. We used a $97.3 \%$ reflectivity OC in a water-cooled mount and found that we could produce up to $2.3 \mathrm{~kW}$ from the laser. This exceeded the IR Demo power record and produced a circulating power of $84 \mathrm{~kW}$.

Using an OC with an even lower loss and a reflectivity of $92 \%$ we were able to push the power up to $4.1 \mathrm{~kW}$. At this power level the FEL itself becomes a good diagnostic for measuring mirror absorption. This allowed us to tune the wavelength for minimum loss. We found that the loss for the output coupler at 5.75 microns was only 250 parts per million (PPM). The high reflector had a loss of 400 PPM including an estimated 100 PPM of substrate transmission absorbed on the cooling backplate.

One hypothesis for the high losses in the downstream mirror was that $\mathrm{THz}$ edge emission from the dipole just upstream of the mirror might dominate the OC heating. Measurements at an electron-beam energy of $80 \mathrm{MeV}$ indicated that this was not the case. However, at an electron beam energy of 145 $\mathrm{MeV}$ the absorbed $\mathrm{THz}$ power went up dramatically, ranging from 50-85 W at $5 \mathrm{~mA}$ depending on details of the micropulse length. The maximum allowed power in the output coupler at 6 microns is 30 $\mathrm{W}$. The absorbed $\mathrm{THz}$ power would not allow us to run at $10 \mathrm{~kW} \mathrm{CW}$, though we could run $10 \mathrm{~kW}$ with up to a $30 \%$ duty cycle and could run $\mathrm{CW}$ with over $6 \mathrm{~kW}$ of power output. 
To avoid THz heating of the output coupler we reversed the optical cavity so that the backplanecooled high reflector, which can absorb over $200 \mathrm{~W}$ of power before limiting laser power, was downstream. The power absorbed in the output coupler was now just the fundamental power. The power absorbed in the high reflector consisted of three sources: the absorbed fundamental power, coherent second harmonic power, and THz edge radiation. This configuration allowed us to run $10 \mathrm{~kW}$ with a duty cycle of over $50 \%$ and to run $\mathrm{CW}$ with up to $8.5 \mathrm{~kW}$ of laser output power.

Since the time constant for mirror distortion is quite long it proved possible to run for periods of up to 1 second with power exceeding $10 \mathrm{~kW}$. When shorter pulses were run the power was as high as 11 $\mathrm{kW}$ during 0.25 second pulses. The macropulse power vs. pulse length is shown in figure 5 illustrating the impact of mirror heating leading to optical distortions. The $8.6 \mathrm{~kW}$ value achieved at 1.5 seconds did not further degrade: hours of operation at this level were achieved.

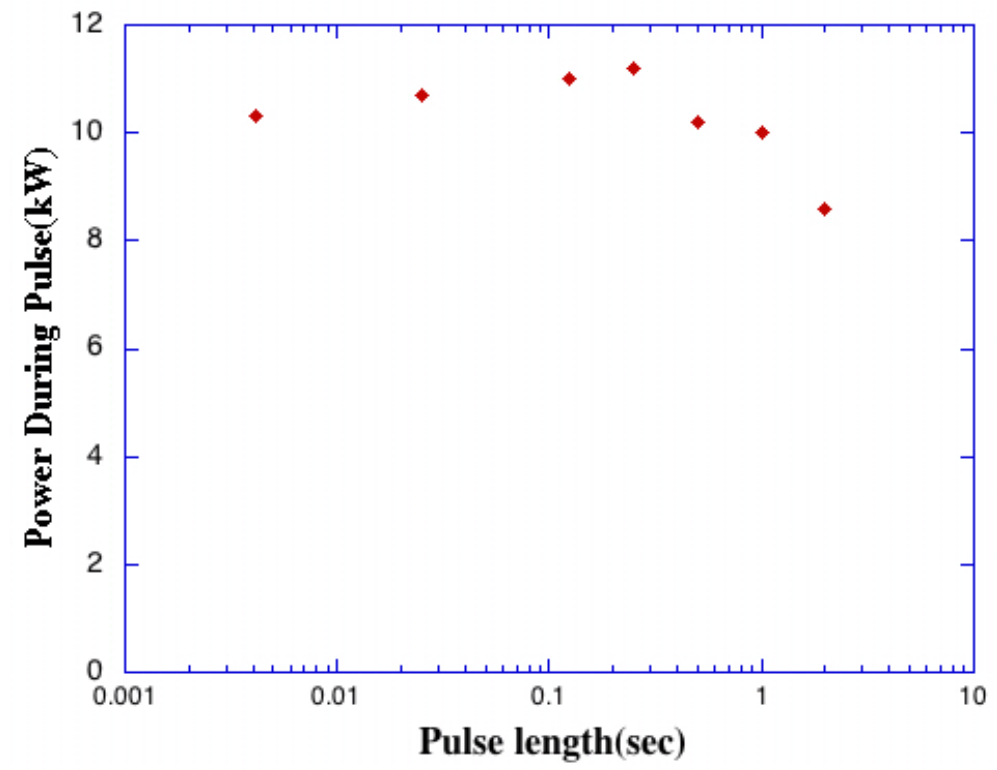

Figure 5. Burst averaged power as a function of pulse length. The point at 0.5 seconds was not fully optimized. The power was calculated assuming negligible turn-on time. The rise in power with pulse length is partly due to this and partly due to the fact that the high reflector radius of curvature was optimized for the longer pulses.

When lasing at 5.75 microns and $10 \mathrm{~kW}$ the output coupling was $8 \%$. This means that the circulating power exceeded $125 \mathrm{~kW}$. Even scatter of at the parts per thousand level can lead to major problems in the cavity. We found that the rings holding the mirrors tended to heat after a time running at high power. This led to changes in the mirror alignment and cavity length. We had planned to use a helium-neon laser to track the mirror angle. Scattered light absorbed in the windows for this system distorted them sufficiently so that this was not possible. When we tried to shield the windows with metal screens we found that the scattered light melted holes in them. THz light also heated beam line elements and led to pressure excursions and optical distortion. Clearly, high power laser systems have to account for all power losses in the system and must be shielded from spurious light of all wavelengths. We now have water cooled optical shields around key components to deal with this.

For the optical resonator we have developed a technology that allows us to change the radius of curvature of a backplane cooled mirror while holding the mirror figure to better than $1 / 20^{\text {th }}$ of a wave. 
We plan to use this type of mirror for the high reflector of the resonator. This means that it is possible to keep the Rayleigh range constant as the output coupler heats up. The power limit is now set by the induced aberration, waist movement, and the intensity on the mirrors. These can all lead to a saturation of the FEL output power.

When the power output of an FEL is several kilowatts in the mid-infrared even measuring the power is a challenge. We found that calcium fluoride windows could not handle transmitted power exceeding $1500 \mathrm{~W}$ at 6 microns. We therefore moved to an in vacuo power meter designed and built at Jefferson Lab capable of handling up to $50 \mathrm{~kW}$ of laser power. The power is absorbed in a black copper coating in a water-cooled cone. The rise in the water temperature of the cooling water and the flow are monitored and used to calculate the absorbed power. It is possible that power is also lost due to backscatter and conduction so the power numbers quoted here are lower limits. Comparison at low power with a commercial power meter indicated that the power reading might be as much as $10 \%$ low, though the uncertainty was large in this calibration.

\section{APPLICATIONS}

We anticipate an exciting and productive program of user experiments starting this year on the Upgrade in the same manner of operation as the original IR Demo activities. Approximately $70 \%$ of the FEL power was delivered to user labs for application experiments. Our operational efforts focused on providing this light for a range of scientific and industrial applications [13-16] and using the machine to explore accelerator and FEL physics issues, especially those relevant to our planned upgrade to $10 \mathrm{~kW}$ output power at $1 \mu \mathrm{m}$.

The JLab IR Demo FEL enabled a rich applied and basic science program that included the investigation of both linear and nonlinear phenomena in proteins, ceramics, and metals, pulsed laser ablation and deposition, laser nitriding, synthesizing carbon nanotubes and micromachining. Linear dynamics using pump-probe techniques was applied to amide-I absorption at 5 to $8 \mu \mathrm{m}$ in proteins [17] and to hydrogen defects in silicon at $3 \mu \mathrm{m}[18,19]$. In the following we expand on some of these applications of a high power, high repetition rate IR FEL.

Pulsed laser ablation and deposition (PLD) is a potential application for manufacturing large-area films. The FEL's ultra fast pulses offer a low ablation threshold, substantially lessened target damage, and particulate elimination. High repetition rate implies high deposition rate, and may mean greater control over ablation and growth dynamics. Wavelength tunability results in enhanced ablation and deposition with resonant absorption, such as in polymers, using specific resonances to control growth processes. We have demonstrated PLD of metals such as niobium and steel [20] We also have performed substantial work on PLD of polymers [21].

Laser nitriding is a method of modifying the properties of metals to obtain a harder surface with better corrosion resistance and the ability to hold higher standoff voltages. The JLab FEL was successfully used to produce high-quality nitride films on iron, titanium, and silicon [22]. Most interestingly the work showed successful nitriding could be achieved at atmospheric pressure without resort to a vacuum chamber. This implies much lower process costs in an industrial setting.

Carbon nanotube structures present a range of production challenges that might be circumvented or better understood by use of the JLab FEL's repetition rate, wavelength tuning and power. Studies were performed to determine how the structures are formed to discover optimum conditions for making them with tailored properties, as well as to learn more about real-time process monitoring and control. The JLab FEL with $3 \mu \mathrm{m}$ light at 400 to $600 \mathrm{~W}$ average power synthesized single-wall carbon nanotubes with smaller diameters than nanotubes produced by direct current arc or table-top pulsed laser vaporization. Production rates were measured in milligrams per minute rather than milligrams per hour [23].

Micromachining is applied to metals for automotive engine applications, and on glasses and ceramics for the fabrication of truly three-dimensional microstructures. With nano-scale engineering of 
key features, it is hoped that satellites could be reduced to the size of baseballs or smaller [24]. We intend to explore the development of this application more extensively using the output of the UV Upgrade presently under construction. Production rates should be significantly enhanced over available excimer laser systems and the range of materials that can be employed will be greatly expanded. We are installing this year a laser microengineering tool to permit CAD control of the laser beam to directly micromachine objects up to $30 \mathrm{~cm}$ x $30 \mathrm{~cm}$ x $50 \mathrm{~cm}$ with a resolution better than 10 microns.

The capabilities of the Upgrade facility in all its operational bands exceed what is available from any other source in the world and we invite prospective users of the photons to review our website at www.jlab.org/FEL or contact us for more information.

\section{ACKNOWLEDGEMENT}

This work was supported by U.S. DOE Contract No. DE-AC05-84-ER40150, the Office of Naval Research, the Air Force Research Laboratory, the Army Night Vision Laboratory, the Commonwealth of Virginia and the Laser Processing Consortium.

\section{REFERENCES}

1. Carr, et al., Nature 420, 153-156 (2002).

2. Neil, et al., Phys. Rev. Lett. 84, 662-665 (2000).

3. D. R. Douglas, et al., Proc. Linac 2000, Monterey, August 21-25, 2000.

4. S. V. Benson, G. Biallas, J. Boyce, D. Douglas, H. F. Dylla, R. Evans, A. Grippo, J. Gubeli, K. Jordan, G. Krafft, R. Li, J. Mammosser, L. Merminga, G. R. Neil, L. Phillips, J. Preble, M. Shinn, T. Siggins, R. Walker, and B. Yunn, Proceedings: 2001 Particle Accelerator Conference, Peter W. Lucas, Sara Webber, editors, IEEE, Piscataway, NJ, 2001.

5. Tim Siggins, Charles Sinclair, Court Bohn, Donald Bullard, David Douglas, Al Grippo, Joe Gubeli, Geoffrey A Krafft, Byung Yunn, Nucl. Instr. And Meth., A475, 549 (2001).

6. J. R. Delayen et al., PAC'99, pp. 934-6, New York, 29 March-2 April, 1999.

7. J. Flanz et al., Nucl. Inst. Meth. A241:325-33 (1985).

8. S. Benson, Nucl. Inst. And Meth. In Phys. Res., A507, 40-43 (2003).

9. S. Benson, Proc. $23^{\text {rd }}$ Int'l FEL Conf., Darmstadt, Germany, August 20-24, 2001. To be published in Nucl. Instr. and Meth.

10. G. R. Neil, et al., Phys. Rev. Letter, 87, 84801 (2001).

11. J.R. Boyce, "Intra-cavity Thomson Scattering," Section 2.5.2, Femtosecond Beam Science, ed. M. Uesaka, World Scientific, In Press.

12. S.Benson, Michelle Shinn, and G. R. Neil, Nucl. Instr. and Meth. A475 531 (2001).

13 H. F. Dylla, Laser Focus World, August 2001.

14. M. J. Kelley, et al, SPIE Int'1. Society for Optical Eng., Issue 2703 pp. 15-20 (1996).

15. H. F. Dylla, SPIE Vol. 3618 (1999).

16. H. F. Dylla et al., SPIE Vol. 3925 40-49, (2000).

17. R. Austin, Phys. Rev. Letter 84, 5435 (2000).

18. M. Budde et al., Phys. Rev. Letter 85, 1452 (2000).

19. G. Lupke, X. Zhang, B. Sun, A. Fraser, N. H. Tolk, and L. C. Feldman, Phys. Rev. Lett. 88, 135501 (2002).

20. M. Shinn, SPIE Vol. 4065, 434-440 (2000).

21. Michael J. Kelley in D. Kumar et al. (Eds.), Laser-Solid Interactions for Materials Processing Proc. Mat. Res. Soc. Symp., 617 (2000).

22. E. Carpene and P. Schaaf, Mat. Res. Soc. Symp. Proc. 780, Y5.8.1 (2003). 
23. P. C. Eklund, B. K. Pradhan, U. J. Kim, Q. Xiong, J. E. Fischer, A. D. Friedman, B. C. Holloway, K. Jordan and M. W. Smith Nano Letters 2, 561 (2002).

24. H. Helvajian (Ed.), Microengineering Aerospace Systems, AIAA, Reston, VA (1999). 